Research report

\title{
Compensatory postural adjustments in Parkinson's disease assessed via a virtual reality environment
}

\author{
Darya Yelshyna $^{a}$, Miguel F. Gago ${ }^{\mathrm{b}, \mathrm{c}, \mathrm{d}, *}$, Estela Bicho ${ }^{\mathrm{a}}$, Vítor Fernandes ${ }^{\mathrm{a}}$, Nuno F. Gago ${ }^{\mathrm{a}}$, \\ Luís Costa ${ }^{a}$, Hélder Silva ${ }^{a}$, Maria Lurdes Rodrigues ${ }^{b}$, Luís Rocha ${ }^{a}$, Nuno Sousa ${ }^{c, d}$ \\ a Algoritmi Center, Department of Industrial Electronics, School of Engineering, University of Minho, Braga, Portugal \\ b Neurology Department, Centro Hospitalar do Alto Ave, EPE, Guimarães, Portugal \\ ${ }^{c}$ Life and Health Sciences Research Institute (ICVS), School of Health Sciences, University of Minho, Braga, Portugal \\ d ICVS-3Bs PT Government Associate Laboratory, Braga/Guimarães, Portugal
}

\section{H I G H L I G H T S}

- Virtual reality allows the analysis of compensatory postural adjustments (CPA).

- IPD patients are highly susceptible to visually induced destabilization.

- CPA is modulated by mechanisms related to different time scales.

- Levodopa treatment increases the stabilizing effect by means of low frequencies CPA

\section{A R T I C L E I N F O}

\section{Article history:}

Received 12 May 2015

Received in revised form 7 July 2015

Accepted 17 August 2015

Available online 21 August 2015

\section{Keywords:}

Idiopathic Parkinson's disease

Compensatory postural adjustments

Virtual reality

Kinematics

Time-frequency distribution

\begin{abstract}
A B S T R A C T
Postural control is a complex dynamic mechanism, which integrates information from visual, vestibular and somatosensory systems. Idiopathic Parkinson's disease (IPD) patients are unable to produce appropriate reflexive responses to changing environmental conditions. Still, it is controversial what is due to voluntary or involuntary postural control, even less what is the effect of levodopa. We aimed to evaluate compensatory postural adjustments (CPA), with kinematic and time-frequency analyzes, and further understand the role of dopaminergic medication on these processes. 19 healthy subjects (Controls) and 15 idiopathic Parkinson's disease (IPD) patients in the OFF and ON medication states, wearing IMUs, were submitted to a virtual reality scenario with visual downward displacements on a staircase. We also hypothesized if CPA would involve mechanisms occurring in distinct time scales. We subsequently analyzed postural adjustments on two frequency bands: low components between 0.3 and $1.5 \mathrm{~Hz}(\mathrm{LB})$, and high components between 1.5 and $3.5 \mathrm{~Hz}(\mathrm{HB})$. Vertical acceleration demonstrated a greater power for discriminating IPD patients from healthy subjects. Visual perturbation significantly increased the power of the HB in all groups, being particularly more evident in the OFF state. Levodopa significantly increased their basal power taking place on the LB. However, controls and IPD patients in the ON state revealed a similar trend of the control mechanism. Results indicate an improvement in muscular stiffness provided by levodopa. They also suggest the role of different compensatory postural adjustment patterns, with LB being related to inertial properties of the oscillating mass and $\mathrm{HB}$ representing reactions to the ongoing visual input-changing scenario.
\end{abstract}

(C) 2015 Elsevier B.V. All rights reserved.

\section{Introduction}

Postural control is a complex dynamic mechanism, which integrates information from visual, vestibular and somatosen-

\footnotetext{
* Corresponding author at: Neurology Department, Centro Hospitalar do Alto Ave, Rua dos Cutileiros, Creixomil. 4835-044 Guimarães, Portugal.

E-mail address: miguelfgago@yahoo.com (M.F. Gago).
}

sory systems, with the central nervous system (brain and spinal cord) adapted to the musculoskeletal system status (muscles, bones, tendons and ligaments). Even during quiet standing, beside the mechanical antero-posterior, ankle strategy, and the mediallateral hip strategy, the center of mass (COM) is also continuously controlled by a central nervous system (CNS) time-delayed feedback loop, in reaction to predictable and unpredictabe postural perturbations [1]. Anticipatory (APA) and compensatory postural adjustment (CPA) strategies are the two main mechanisms used 
by the CNS in order to deal with body perturbations that may either be internally generated (e.g., self-initiated movements) or externally generated (e.g., being pushed at shoulder level while walking) [2]. When postural perturbation is unpredictable, postural muscles are activated to restore stability after the moment of perturbation. These later responses (CPA) are triggered by sensory feedback signals and help in dealing with the actual effects of a perturbation $[3,4]$. While APA are observed only in the case of predictable perturbations, CPA are seen during both, predictable (following APA) and unpredictable perturbations. Often CPA are of larger magnitude in response to unpredictable perturbations [5].

The body schema is a perception of one's body in space and body parts associated with movement and is influenced by visuomotor processing. The temporoparietal cortex, including the posterior parietal cortex and the vestibular cortex, appears to integrate realtime signals in the visual, proprioceptive, and vestibular sensations so that the body schema can be always updated. [6]. Absence or degradation of any type of sensory input may affect balance performance. The latter is commonly observed with aging, leading to increased instability, falls and consequent injuries [7-9]. Disorders such as Idiopathic Parkinson Disease (IPD) further aggravate balance disturbances of elderly population [10,11]. Deficiency in the information processing from the temporoparietal cortex to the frontal cortex may cause errors in APA, CPA and gait difficulties, such as the "freezing of gait" [6].

Postural control is compromised in subjects with untreated IPD due to disturbed postural reflexes, poor control of voluntary movements, orthostatic hypotension and side effects of certain medications that include dyskinesia [12]. Compared to healthy elderly adults, IPD patients are unable to produce appropriate reflexive responses to changing environmental conditions. In IPD, besides motor deficits contribution, postural impairment is also associated with abnormal spatial and temporal processing of sensory information, producing incorrect signals for the preparation and execution of voluntary movement $[11,13]$. It has been established that APA and CPA are compromised in IPD, with patients not only having difficulty switching between postural strategies, but also being unable to appropriately scale the size of their postural responses to the size of environmental change [14-17]. Discrepancies of reports, with IPD patients presenting larger or decreased sway, are probably due to differences in voluntary and involuntary posture postural control and/or study design [18]. Moreover, effects of levodopa treatment are controversial. Some studies found a worsening of sway abnormalities in view of increased sway area and reduced mean velocity $[19,20]$, while others report a larger and a faster sway [21,22]. Regarding CPAs, levodopa has been reported not to improve slower CPAs on IPD patients and theirs difficulty in using cognitive set to modify responses to surface perturbations [15]. In fact, levodopa therapy can compromise the immediate postural adaptation and refinement of postural strategy, changes in amplitude of vertical ground reaction forces and forces applied to support apparatus within conditions between the initial and final trials, that is present on the OFF state and on healthy subjects [23].

In the last years, the impact of visual perturbation on postural adjustments has been widely explored. Visual deprivation during quiet stance eliminates one of the inputs to the control mechanism, producing a destabilizing effect. This process results in an increasing need for postural adjustments, affecting in a larger scale elderly impaired subjects $[8,24,25]$ Susceptibility to visual stimulation has been studied in several conditions, such as visual focus on differently distanced targets [26] or exposal to a moving surround, which in most recent researches, was implemented through an immersive virtual scene allowing for a better perception of the induced motion. This virtual reality creates an illusion that puts the subject in a different place other than where she/he physically is [27]. Besides inducing self-motion illusion, moving visual surround conflicts with perceptions from somatosensory and vestibular systems, since the body has not actually moved. As a consequence, the body generates CPA in the direction of the visual perturbation [28,29].

Balance integrity is most commonly assessed by kinetic and kinematic analyzes [30]. Estimates of center of pressure (COP) trajectory derived from force platforms have been extensively used to compute stationary sway measures, presented in time and frequency domains [7]. Recently, accelerometry emerged as an alternative technique to posturography, successfully exploiting the same measures with the advantages of lower cost, reduced size and portability. Inertial Measurement Units (IMUs), which include both accelerometers and gyroscopes, provide additional information about body tilt and orientation [31,32]. Stationary signal analysis provides insightful information regarding the postural control. However, it has long been demonstrated that this system is dynamically regulated [33]. An appropriate non-stationary technique should be employed to characterize the existing spectral variations, such as time-frequency analysis [34] . Interestingly, numerous studies have also reported that these changes occur in two distinct time scales: a fast (high frequency) open-loop control and a slower (low frequency) corrective feedback-based control $[1,35]$.

The primary objective of this research is to evaluate visually induced CPA in a changing virtual reality scenario, in healthy subjects (controls) and IPD patients, by means of kinematic and time-frequency analyzes of IMU records. As a second objective, we aim to understand the role of dopaminergic medication on postural adjustment mechanisms.

\section{Methodology}

\subsection{Subjects and clinical assessment}

The study protocol was derived from the ICVS-3Bs and the Algoritmi Center and was approved by the hospital local ethics committee. Written informed consent was received from all participants in the study. 15 patients and 19 controls were consecutively recruited from our Movement Disorders outpatient consult, fulfilling criteria for IPD (UKPDS Brain Bank criteria). IPD patients had normal clinical postural stability measured by the retropulsion test (item 12 on MDS-UPDRS-III), had an Hoehn \&Yahr of 2 (OFF state). The exclusion criteria were dementia, orthopedic, musculoskeletal, vestibular disorder, significant auditory deficit, and alcohol abuse. Patients had no somatosensory deficit, on neurological examination, nor wore glasses or contact lens to correct vision. The collected variables consisted of demographic (gender, age, and education) and biometric data reported as influencing kinetic performance, such as weight, height, body mass index. Clinical data were also collected, including years of disease duration, Movement Disorder Society-Unified Parkinson's disease rating scale III (MDS-UPDRS III) (scored as either an in the OFF or in the ON state), levodopa equivalent daily dose [36], and morning levodopa challenge dose. A brief neuropsychological examination was performed using the Portuguese version of the Montreal Cognitive Assessment test (MoCA) with scores normalized to the Portuguese population [37] no more than 1 month prior to the kinetic assessment. The levels of education were categorized by years of schooling as follows: 0 (analphabetic), 1 ( $1-4$ years), 2 (5-9 years), 3 (10-12 years), and 4 (>12 years). 


\subsection{Apparatus and postural tasks}

One sensing module, harboring an 8051 microprocessor embedded in CC2530 Texas Instrument SoC (System on Chip), and a wearable inertial measurement unit MPU6000 (tri-axial accelerometer and gyroscope), operating with a sample rate frequency of $118 \mathrm{~Hz}$ on a SD card [38], was attached to the center of mass (COM), located at $55 \%$ of the patient's height above the ground.

The subjects were submitted to a realistic visual scene with three-dimensional depth information by wearing the Oculus Rift goggles - a virtual reality headset device with a $100^{\circ}$ field-ofview. Tuscany Demo (Fig. 1) was chosen as the virtual scenario for performing unexpected visually induced motion on a staircase and evaluation of the response to the illusory perception of falling (translation down the stairs). While performing the tasks, the subjects were instructed to maintain still standing, barefoot, with the medial aspects of the feet touching each other, arms hanging at their sides and using a safety ceiling trunk belt. Subjects were instructed not to abandon this position and, if need be, to do a corrective adjustment by bending their knees. During the preparatory phase, subjects were asked to identify and search for several objects embedded in the VR setting (e.g., floor, roof, stairs, fireplace, chandelier, window and door). Visual focus and eye width settings were adjusted for each participant to display a clear stereoscopic 3-D image. Initially, the subjects stood at the top of the staircase in the virtual environment and were issued to focus their attention on the first stairs. Approximately ten seconds later, the scenario instantaneously moved down, creating a visual displacement, translating the subject to the middle of the stairs. After another $10 \mathrm{~s}$, the scenario moved upwards, placing the subject back on the top of the stairs, until the next trial. After experimenting several translations, subjects were asked to explain if they felt that they were virtually pushed into the middle of the stairs, as they would have experienced in real life. This procedure was repeated several times with a minimum 20-s gap between trials and a minimum of 5 preparatory trials per subject, as to guarantee immersion into the virtual reality, to a maximum of 8 (in two patients), as assessed by subjects' answer to VR subjective immersion feeling. The neurologist was responsible for triggering the downward translation in a manual and random fashion, thus adverting adaptation and learning effect and putting individuals unaware of the exact moment of the upcoming event. A total of 5 effective "falling" trials per subject, $10 \mathrm{~s}$ duration, with the same methodology as in the preparatory phase, were selected as object of subsequent analysis. The translation in the virtual environment corresponds to approximately 1.17 meters of displacement along the vertical axis.

Patients were clinically and kinematically evaluated in the OFF state, and in the ON state after morning levodopa dose, on the same day. OFF state evaluation took place in the morning, after a $12 \mathrm{~h}$ period without any dopaminergic medication. Afterward, IPD patients were given their usual morning dose dopaminergic medication and were re-examined 90 min later regardless of motor response.

\subsection{Data analysis}

\subsubsection{Kinematic analysis}

IMUs allow for a more elaborate study of motor patterns than procedures relying solely on accelerometers or force platforms. Joining accelerometer and gyroscope information provides us with roll and pitch angles of body orientation, and a reasonable estimation of its position [39] .

No clear procedures have yet been established for accelerometer data processing, but the main energy content of human movement is held below $3.5 \mathrm{~Hz}[30,31]$. For this reason, the raw acceleration signals were filtered with a zero-phase low-pass Butterworth filter with a $3.5 \mathrm{~Hz}$ cutoff frequency. Due to the abdominal placement of the sensor, acceleration signals went through additional high-pass filtering in order to eliminate interference that might be caused by the act of breathing. Considering a normal respiratory frequency in adults, i.e., 18 breaths per minute [40], we applied a zero-phase high-pass Butterworth filter with a $0.3 \mathrm{~Hz}$ cutoff frequency.

Another version of the accelerometer signal - one that takes into account lower frequency components - should be applied in order to characterize body orientation and approximate its displacement $[24,32]$. For this purpose, both the raw acceleration and gyroscope signals underwent zero-phase low-pass Butterworth filtering with $0.5 \mathrm{~Hz}$ and $2 \mathrm{~Hz}$ cutoff frequencies, respectively. Detailed explanation of the methodology used to obtain body orientation - pitch and roll angles - and estimate COM displacement can be found in our previous study [24].

In order to guarantee an equal level of sensory perturbation, we selected the last trial from each subject as being the most representative of the overall response to the visual motion. Kinematic measures were computed from $8 \mathrm{~s}$ time segments after the last onset of the visual down-moving stimulus. Table 2 contains timedomain features that characterize sway magnitude and variability.

\subsubsection{Time-frequency analysis}

Many real-world signals, such as postural adjustment recordings, are non-stationary, implying that their spectral content changes over time. This is especially true when trying to assess responses to a particular stimulus. Classical frequency representations based on Fourier transform cannot provide an accurate spectrum analysis, since they only reflect global frequency content without specifying when the changes occurred. The concept of time-frequency distributions (TFD) was introduced to circumvent this limitation, as functions in both time and frequency [41].

One of the first presented solutions implements an extension to traditional Fourier analysis: short-time Fourier transform (STFT). The STFT estimates the energy spectrum (spectrogram) using a sliding window, thus assuming local stationarity of the signal. The resulting spectrogram depends on window size, which is responsible for the trade-off between time and frequency resolutions. The bigger the window size, the better the frequency resolution and the ability to detect low-frequency components that are characteristic to postural adjustments. On the other hand, it sacrifices a large amount of time information, which is especially critical when analyzing short-duration signals. This is the major limitation of spectrograms, making them unsuitable for COM data analysis $[34,42]$.

The minimum mean cross-entropy (MMCE) method solves this problem by combining information from several sources. Given a finite set of spectrograms computed with different window sizes, the resulting TFD is a much better approximation of the time-varying spectrum than any of these individual spectrograms. Investigations on the MMCE method pointed out its low computational demand and ability to closely approximate to positive TFDs of the Cohen-Posch class, which are the most appropriate for realworld signal analysis $[43,44]$.

The majority of studies concerning postural adjustments evaluation using inertial sensors considered the vertical component of acceleration insignificant and restricted their analysis to the 2D plane - a common practice in posturography. If a vertical visual motion is evaluated, these data might not be able to properly reflect posture responses [27]. A study, which evaluated outputs from all accelerometers' axis to detect age-related changes in postural balance, detected a great sensitivity of the vertical component for distinguishing young from elderly subjects [45]. This interesting finding, along with the direction of our VR visual stimulus, and also the displacement occurring mainly on the z-axis (Table 2), led to the selection of vertical acceleration for time-frequency analysis. 


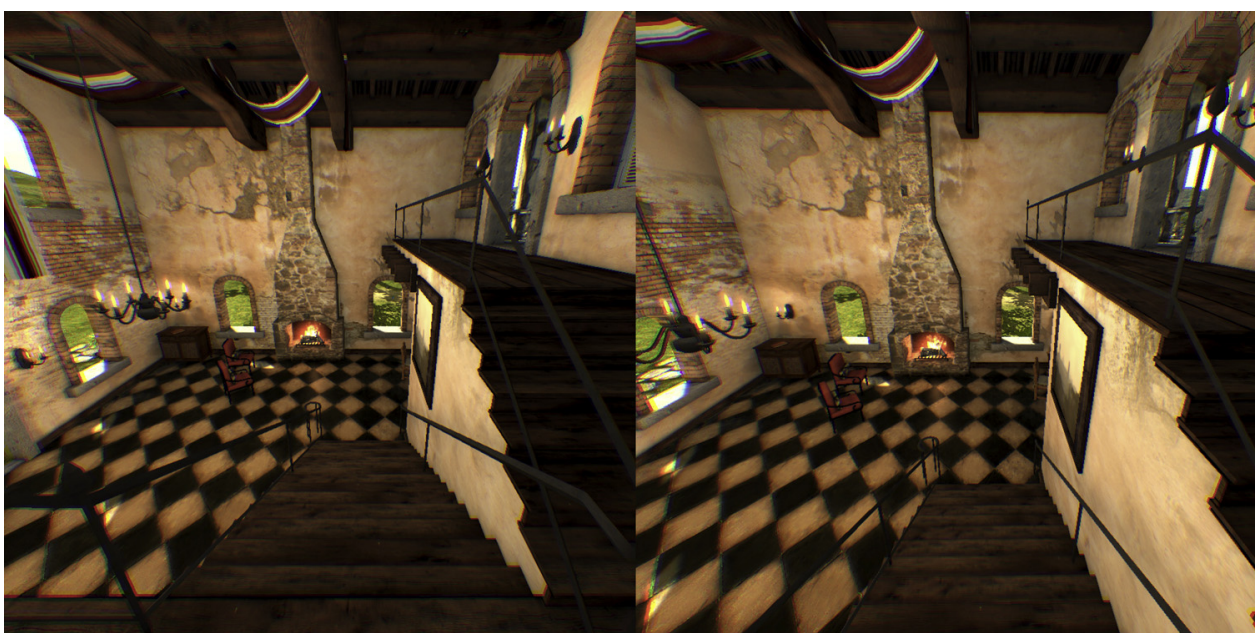

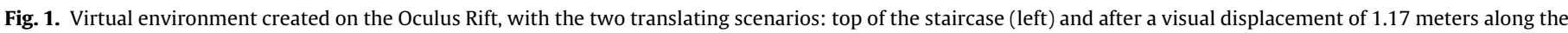
vertical axis, translating to the middle of the stairs (right).

Table 1

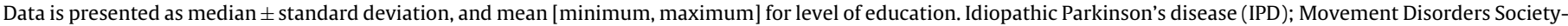

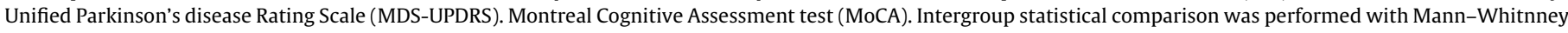
test.

\begin{tabular}{|c|c|c|c|}
\hline & Controls $(n=19)$ & $\operatorname{IPD}(n=15)$ & Inter-group comparison \\
\hline Gender (female/male) & $8 / 11$ & $9 / 6$ & $* p=0.491$ \\
\hline Age & $69.9 \pm 6.7$ & $71.6 \pm 8.1$ & $U=123.5 ; z=-0.662 ; p=0.519$ \\
\hline Weight (kg) & $74.9 \pm 12.6$ & $73.5 \pm 12.1$ & $U=140 ; z=-0.087 ; p=0.939$ \\
\hline Height $(\mathrm{m})$ & $1.63 \pm 0.09$ & $1.61 \pm 1.0$ & $U=124 ; z=-0.625 ; p=0.542$ \\
\hline Body Mass Index (Kg/m2) & $28.0 \pm 2.6$ & $28.1 \pm 2.7$ & $U=133 ; z=-0.330 ; p=0.758$ \\
\hline Level of Education & $1[0,2]$ & $1[0,1]$ & $\chi^{2}=2.359 ; p=0.456$ \\
\hline MoCA & $24.2 \pm 3.3$ & $22.2 \pm 3.7$ & $U=95 ; z=-1.656 ; p=0.10$ \\
\hline Disease duration (years) & . & $5.3 \pm 2.6$ & \\
\hline MDS-UPDRS III Off stage & . & $43.7 \pm 15.0$ & \\
\hline MDS-UPDRS III On stage & . & $18.3 \pm 9.5$ & \\
\hline Levodopa Equivalent Daily Dose & . & $1108 \pm 434$ & \\
\hline Levodopa morning dose & . & $389 \pm 178$ & \\
\hline Motor Benefit (\%) & . & $59.1 \pm 14.5$ & \\
\hline
\end{tabular}

Generally, a TFD by itself provides mainly descriptive information. A quantitative description of its dynamic behavior can be achieved by defining several time-dependent parameters: instantaneous average power (IAP), instantaneous mean frequency (IMF) and instantaneous bandwidth (IBW), also known as standard deviation of frequency content [46,47]. The IAP allows monitoring sudden changes due to postural adjustments. The IMF represents the overall frequency shift at each time.

After being downsampled to $10 \mathrm{~Hz}$, the vertical acceleration assessed the dynamical properties of the CPAs. For each subject, we extracted time segments including all trials with a margin of $10 \mathrm{~s}$ prior to the first and after the last stimulus. Time-varying spectrum was estimated by an MMCE combination of spectrograms computed with Hanning windows of 15, 31 and 127 samples [43]. The Time-Frequency Toolbox (v1.2) for MATLABC provided the function for MMCE computation.

On a subsequent analysis, we hypothesized that postural adjustments may involve mechanisms occurring in distinct time scales and thus two frequency bands were defined: a lower band containing components between 0.3 and $1.5 \mathrm{~Hz}(\mathrm{LB})$, and a higher band with components between 1.5 and $3.5 \mathrm{~Hz}(\mathrm{HB})$. A more precise evaluation of the visual destabilization is achieved when the subject is focused and unaware of the upcoming event. In order to identify this increased attentional demand during quiet stance, we computed the total power of the high-frequency band $4 \mathrm{~s}$ prior to the visual stimulus for all trials, and selected the one with the lowest value. All data processing and feature computation were performed with a custom-made MATLABC code.

\subsection{Statistical analysis}

Gender comparisons were analyzed by the $\chi^{2}$ Fisher exact test. Given the small number of subjects, the intergroup statistical analysis was carried out with a non-parametric Mann-Whitney exact test. Wilcoxon matched pair test was used for Control and IPD's intragroup changes, for separate band powers of TFD analysis, and for the evaluation of levodopa treatment. Statistical analyzes were conducted with software (SPSS 20.0) using a 95\% level of significance.

\section{Results}

\subsection{Subjects}

Fifteen patients with IPD (6 women, 9 men) and nineteen controls (11 females/8 males) were included in this study. The demographic and anthropometric characteristics of the two groups are summarized in Table 1 , as is the clinical characteristics of IPD patients. Groups were equally matched for demographic and anthropometric characteristics. IPD patients had a positive response to morning levodopa dose of $59.1 \%$ (mean), 14.5 (standard 
Table 2

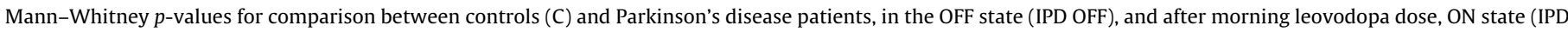
$\mathrm{ON})$. Significant $p$-values were shadowed. Standard deviation (SD); average acceleration magnitude (AAM).

\begin{tabular}{|c|c|c|c|c|c|c|}
\hline \multirow[b]{2}{*}{ Kinematic parameters } & \multicolumn{3}{|l|}{$($ Mean \pm SD $)$} & \multicolumn{3}{|c|}{ Intergroup comparison ( $p$-value) } \\
\hline & $\mathrm{C}$ & IPD OFF & IPD ON & C vs. IPD OFF & C vs. IPD ON & IPD OFF vs. IPD ON \\
\hline SD velocity $(\mathrm{cm} / \mathrm{s})$ & $0.634 \pm 0.083$ & $0.733 \pm 0.379$ & $0.799 \pm 0.296$ & 0.811 & 0.137 & 0.303 \\
\hline Maximum velocity $(\mathrm{cm} / \mathrm{s})$ & $2.841 \pm 0.385$ & $3.357 \pm 1.647$ & $3.926 \pm 1.697$ & 0.681 & 0.023 & 0.095 \\
\hline SD velocity on X-axis $(\mathrm{cm} / \mathrm{s})$ & $0.959 \pm 0.172$ & $0.991 \pm 0.511$ & $1.055 \pm 0.305$ & 0.537 & 0.372 & 0.330 \\
\hline Maximum velocity on $\mathrm{X}$-axis $(\mathrm{cm} / \mathrm{s})$ & $2.295 \pm 0.442$ & $2.322 \pm 1.163$ & $2.293 \pm 0.633$ & 0.190 & 0.918 & 0.679 \\
\hline SD velocity on Y-axis $(\mathrm{cm} / \mathrm{s})$ & $0.829 \pm 0.237$ & $1.087 \pm 0.584$ & $1.219 \pm 0.737$ & 0.336 & 0.128 & 0.454 \\
\hline Maximum velocity on Y-axis $(\mathrm{cm} / \mathrm{s})$ & $1.963 \pm 0.691$ & $2.668 \pm 1.223$ & $3.146 \pm 2.090$ & 0.128 & 0.060 & 0.359 \\
\hline Sway Path $(\mathrm{cm})$ & $9.016 \pm 1.516$ & $10.509 \pm 5.039$ & $11.828 \pm 4.902$ & 0.758 & 0.137 & 0.252 \\
\hline Sway Area $\left(\mathrm{cm}^{2}\right)$ & $1.959 \pm 0.919$ & $3.747 \pm 5.702$ & $3.270 \pm 2.556$ & 0.271 & 0.451 & 0.934 \\
\hline Elliptic Area $\left(\mathrm{cm}^{2}\right)$ & $4.918 \pm 3.006$ & $8.354 \pm 12.223$ & $7.363 \pm 6.315$ & 0.410 & 0.607 & 0.599 \\
\hline SD acceleration on $\mathrm{X}$-axis $\left(\mathrm{cm} / \mathrm{s}^{2}\right)$ & $0.448 \pm 0.091$ & $0.428 \pm 0.134$ & $0.475 \pm 0.183$ & 0.410 & 0.973 & 0.359 \\
\hline Range acceleration on $\mathrm{X}$-axis $\left(\mathrm{cm} / \mathrm{s}^{2}\right)$ & $2.432 \pm 0.548$ & $2.298 \pm 0.665$ & $2.423 \pm 0.893$ & 0.256 & 0.681 & 0.762 \\
\hline AAM acceleration on $\mathrm{X}$-axis $\left(\mathrm{cm} / \mathrm{s}^{2}\right)$ & $0.346 \pm 0.076$ & $0.337 \pm 0.114$ & $0.380 \pm 0.151$ & 0.560 & 0.732 & 0.303 \\
\hline SD acceleration on Y-axis $\left(\mathrm{cm} / \mathrm{s}^{2}\right)$ & $0.391 \pm 0.103$ & $0.438 \pm 0.172$ & $0.472 \pm 0.245$ & 0.656 & 0.515 & 0.389 \\
\hline Range acceleration on Y-axis $\left(\mathrm{cm} / \mathrm{s}^{2}\right)$ & $2.092 \pm 0.663$ & $2.104 \pm 0.789$ & $2.340 \pm 1.304$ & 0.973 & 1.000 & 0.389 \\
\hline AAM acceleration on $\mathrm{Y}$-axis $\left(\mathrm{cm} / \mathrm{s}^{2}\right)$ & $0.306 \pm 0.084$ & $0.349 \pm 0.137$ & $0.375 \pm 0.196$ & 0.471 & 0.451 & 0.389 \\
\hline SD acceleration on Z-axis $\left(\mathrm{cm} / \mathrm{s}^{2}\right)$ & $0.258 \pm 0.070$ & $0.384 \pm 0.136$ & $0.389 \pm 0.179$ & 0.002 & 0.023 & 0.890 \\
\hline Range acceleration on Z-axis $\left(\mathrm{cm} / \mathrm{s}^{2}\right)$ & $1.364 \pm 0.456$ & $2.002 \pm 0.807$ & $2.046 \pm 0.948$ & 0.021 & 0.030 & 0.890 \\
\hline AAM acceleration on Z-axis $\left(\mathrm{cm} / \mathrm{s}^{2}\right)$ & $0.203 \pm 0.055$ & $0.306 \pm 0.103$ & $0.308 \pm 0.143$ & 0.001 & 0.021 & 0.804 \\
\hline
\end{tabular}

deviation). VR visual perturbation elicited no need for a corrective step on either group.

\subsection{Kinematic features}

Kinematic analysis revealed significant differences in velocity and acceleration between healthy subjects and IPD patients. No statistically significant changes in displacement-based measures were found between IPD patients in the ON and OFF states. Nevertheless, levodopa significantly increased the maximum velocity (C vs. IPD ON state $(U=77.0 ; z=-2.27 ; p=0.023)$ ), without a significant prevalence on either axis. The vertical acceleration signal demonstrated a greater power in discriminating IPD patients from Controls. IPD patients, either in the OFF or ON state, showed a significant increase in average acceleration magnitude (AAM) (C vs. IPD OFF: $(U=50.0 ; z=-3.21 ; p=0.001)$; C vs. IPD ON: $(U=76.0$; $z=-2.31 ; p=0.021)$ ), standard deviation (SD) (C vs. IPD OFF: $(U=54.0 ; z=-3.07 ; p=0.002)$; C vs. IPD ON: $(U=77.0 ; z=-2.27$; $p=0.023)$ ), and range of acceleration (C vs. IPD OFF: $(U=76.0$; $z=-2.31 ; p=0.021)$; C vs. IPD ON: $(U=80.0 ; z=-2.17 ; p=0.03)$, on the z-axis (vertical) acceleration signal (Fig. 2).

\subsection{Time-frequency distributions}

a) Instantaneous mean frequency (IMF)

TFD analysis reflected pronounced differences of the frequency patterns in response to visual perturbation, which were perceived at separate power bands level. Intergroup comparison revealed substantial divergence between Controls and IPD patients, on IMF and low and high power bands. Before the environment visual scenario change ( $-4-0 \mathrm{~s}$ interval) IPD in the ON state were shifted to lower IMF (Fig. 3; Table 3) (IPD ON vs. IPD OFF, $p=0.001$ ). Immediately after and towards the end, IPD patients in the ON state tended for a higher IMF (C vs. IPD ON (0-4 s: $z=-2.029, p=0.043),(4-8 \mathrm{~s}$ : $z=-2.168, p=0.030)$ ). Both Control and IPD in the ON state groups presented a monotonic increase in IMF along all considered time intervals (Fig. 3). In contrast, in the OFF state, IPD patients tended for lower IMF ( 4 to $8 \mathrm{~s}$ interval: C vs. IPD OFF, $p=0.017$ ).

Concerning the intragroup analysis, a pronounced shift to higher frequencies was observed in healthy subjects during the last $4 \mathrm{~s}$ interval ( $-4-0$ vs. $4-8 \mathrm{~s}$ : $z=-2.254, p=0.023$ ). In contrast, IPD patients in the OFF state displayed a trend for lower frequencies after the visual perturbation (Fig. 3), which was mostly perceived

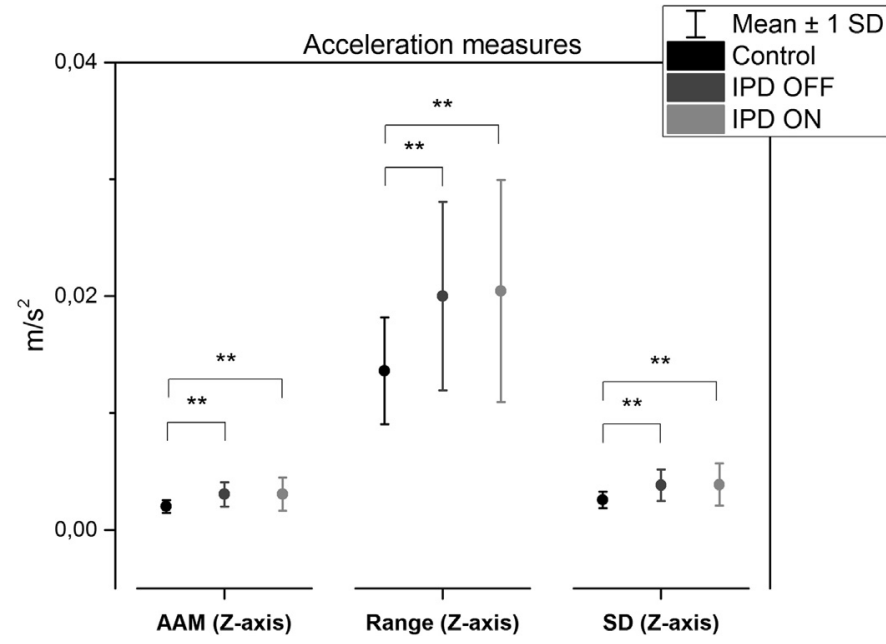

Fig. 2. Mean values of z-axis acceleration measures: average acceleration magnitude (AAM), range and standard deviation (SD) from healthy subjects (control) and IPD patients in the ON (IPD ON) and the OFF (IPD OFF) states. Brackets with two asterisks represent statistically significant $(p<0.05)$ results of intergroup Mann-Whitney test.

at the last $4 \mathrm{~s}(-4-0$ vs. $4-8 \mathrm{~s}:(z=-2.158, p=0.030), 0-4$ vs. $4-8 \mathrm{~s}$; $(z=-1.988, p=0.048))$.

\section{- Lower band (LB) power}

On the LB power, no differences were found between IPD patients in the OFF and ON states. However, OFF state IDP patients exhibited a significantly greater LB than healthy subjects in all of the considered time intervals ( $C$ vs. IPD OFF: $(-4-0 \mathrm{~s}$ : $U=75.0$; $z=-2.34 ; p=0.019)(0-4 \mathrm{~s}: U=53.0 ; z=-3.10 ; p=0.001)(4-8 \mathrm{~s}$ : $U=45.0 ; z=-3.38 ; p<0.001)$ ). Moreover, IPD patients in the ON state showed an even larger increase of LB power in comparison with the Control group (C vs. IPD ON $(-4-0 \mathrm{~s}$ : $U=82.0$; $z=-2.10 ; p=0.036)(0-4 \mathrm{~s}: U=84.0 ; z=2.03 ; p=0.043)(4-8 \mathrm{~s}$ : $U=72.0 ; z=-2.45 ; p=0.014)$ ).

Of note, IPD patients in the ON state and healthy subjects shared the same trend of energy pattern (Fig. 4) in the LB. In contrast, in the OFF state, IPD patients showed an opposite trend, where LB power increased significantly during the post-stimulus condition 
Table 3

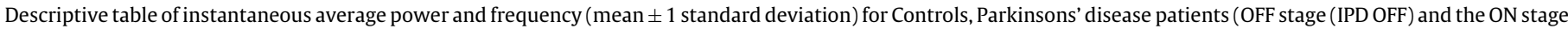

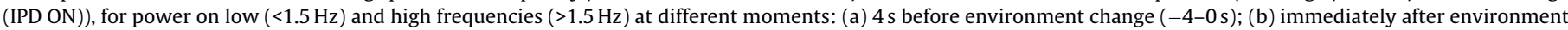

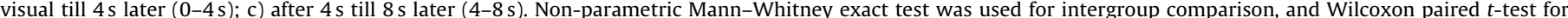
intragroup comparison on different time moments as follows: ( $-4-0 \mathrm{~s})$ vs. (0-4s); (0-4s) vs, (4-8 s); and (-4-0 s) vs. (4-8 s). .Significant $p$-values were shadowed.

\begin{tabular}{|c|c|c|c|c|c|c|c|c|c|}
\hline & \multicolumn{3}{|l|}{ Mean \pm SD } & \multicolumn{3}{|c|}{ Intergroup p-value } & \multicolumn{3}{|c|}{ Intragroup p-value } \\
\hline & C & IPD OFF & IPD ON & $C$ vs IPD OFF & C vs IPD ON & IPD OFF vs IPD ON & C & IPD OFF & IPD ON \\
\hline$-4-0 s$ & & & & & & & -4-0 s vs. 0-4 s & & \\
\hline Mean low power $(\mu)$ & $1.400 \pm 1.487$ & $2.442 \pm 1.915$ & $5.256 \pm 7.883$ & 0.019 & 0.036 & 0.389 & 0.465 & $<0.001$ & 0.107 \\
\hline Mean high power $(\mu)$ & $1.653 \pm 2.216$ & $3.271 \pm 2.427$ & $2.497 \pm 2.744$ & 0.002 & 0.096 & 0.073 & 0.002 & 0.003 & 0.064 \\
\hline Mean IF $(\mathrm{Hz})$ & $1.751 \pm 0.143$ & $1.775 \pm 0.407$ & $1.509 \pm 0.397$ & 0.945 & 0.089 & 0.001 & 0.113 & 0.934 & 0.454 \\
\hline $0-4 s$ & & & & & & & $0-4 \mathrm{~s}$ vs. $4-8 \mathrm{~s}$ & & \\
\hline Mean low power $(\mu)$ & $1.388 \pm 0.844$ & $3.662 \pm 2.756$ & $4.713 \pm 4.577$ & 0.001 & 0.043 & 0.720 & 0.568 & 0.404 & 0.762 \\
\hline Mean high power $(\mu)$ & $2.362 \pm 1.731$ & $4.687 \pm 3.091$ & $3.187 \pm 2.953$ & 0.003 & 0.537 & 0.007 & 0.441 & 0.375 & 0.035 \\
\hline Mean IF $(\mathrm{Hz})$ & $1.826 \pm 0.204$ & $1.763 \pm 0.394$ & $1.564 \pm 0.372$ & 0.864 & 0.043 & 0.064 & 0.134 & 0.048 & 0.121 \\
\hline $4-8 s$ & & & & & & & $-4-0$ vs. $4-8 s$ & & \\
\hline Mean low power $(\mu)$ & $1.320 \pm 0.623$ & $3.809 \pm 2.718$ & $4.185 \pm 3.878$ & $<0.001$ & 0.014 & 0.978 & 0.182 & $<0.001$ & 0.277 \\
\hline Mean high power $(\mu)$ & $3.300 \pm 3.754$ & $4.127 \pm 3.078$ & $5.871 \pm 8.723$ & 0.128 & 0.202 & 0.421 & $<0.001$ & 0.029 & $<0.001$ \\
\hline Mean IF $(\mathrm{Hz})$ & $1.905 \pm 0.226$ & $1.635 \pm 0.387$ & $1.701 \pm 0.303$ & 0.017 & 0.030 & 0.679 & 0.023 & 0.030 & 0.055 \\
\hline
\end{tabular}

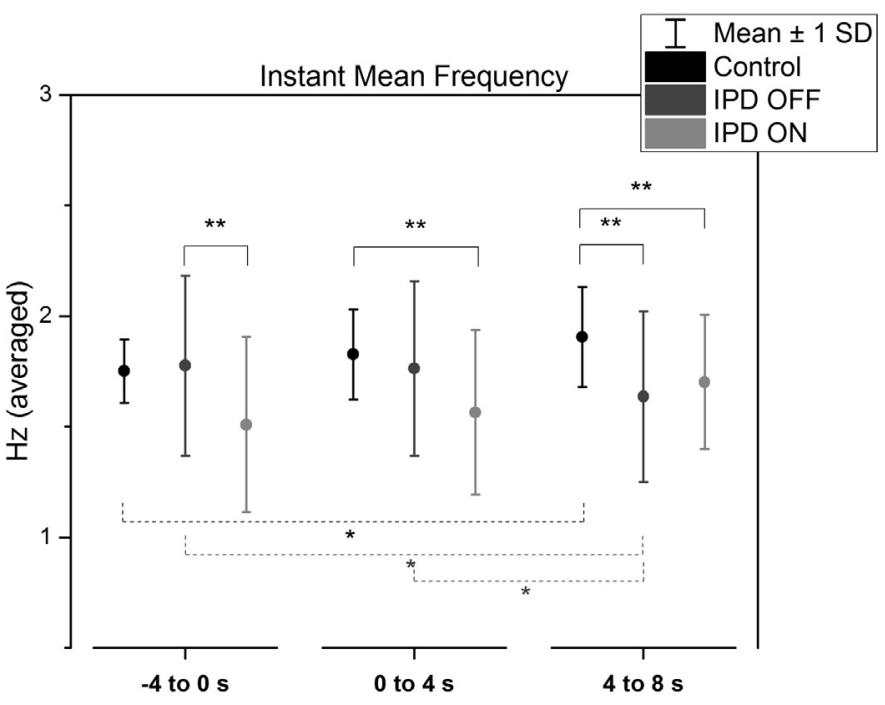

Fig. 3. Mean values of instantaneous mean frequency for healthy subjects (control) and IPD patients (in the OFF (IPD OFF) and ON (IPD ON) stages) averaged over $4 \mathrm{~s}$ intervals. Brackets with two asterisks represent statistically significant $(p<0.05)$ results of intergroup Mann-Whitney test. Brackets with one asterisk and dashed lines represent statistically significant $(p<0.05)$ results of intragroup Wilcoxon paired $t$-test.

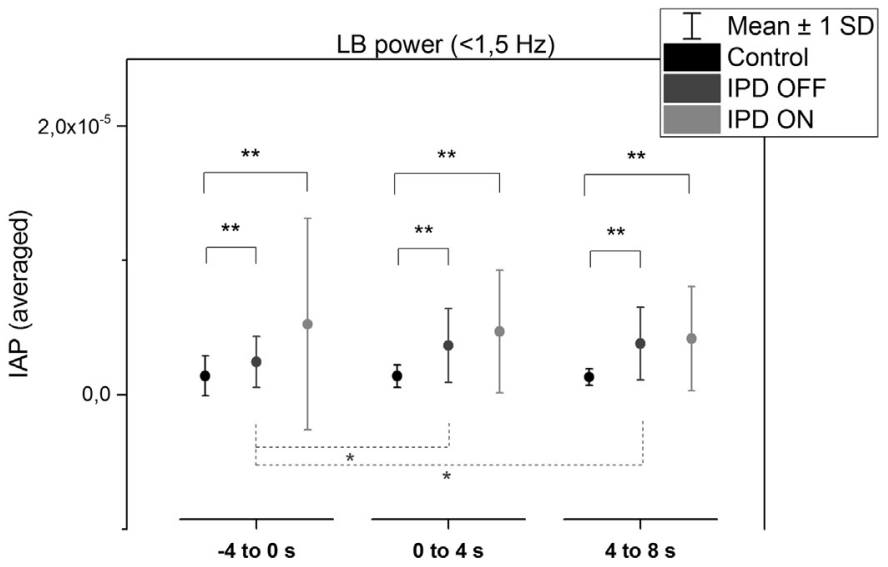

Fig. 4. Mean values of low frequency band power $(<1.5 \mathrm{~Hz}$ ) for healthy subjects (control) and IPD patients (in the OFF (IPD OFF) and ON (IPD ON) stages) averaged over $4 \mathrm{~s}$ intervals. Brackets with two asterisks represent statistically significant $(p<0.05)$ results of intergroup Mann-Whitney test. Brackets with one asterisk and dashed lines represent statistically significant $(p<0.05)$ results of intragroup Wilcoxon paired $t$-test

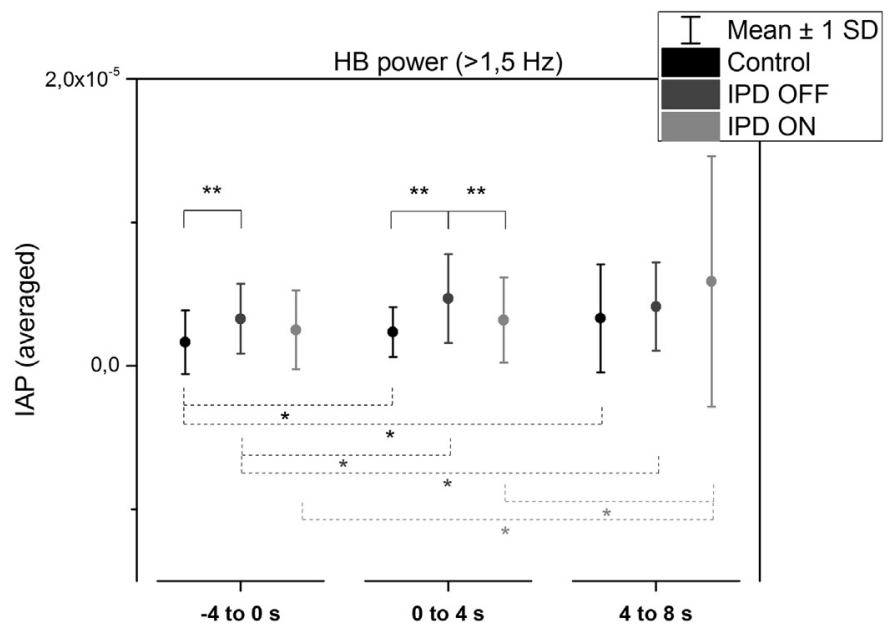

Fig. 5. Mean values of high frequency band power $(>1.5 \mathrm{~Hz})$ for healthy subjects (control) and IPD patients (in the OFF (IPD OFF) and ON (IPD ON) stages) averaged over $4 \mathrm{~s}$ intervals. Brackets with two asterisks represent statistically significant $(p<0.05)$ results of intergroup Mann-Whitney test. Brackets with one asterisk and dashed lines represent statistically significant $(p<0.05)$ results of intragroup Wilcoxon paired $t$-test.

$(-4-0$ s vs. $0-4$ s $(z=-3.258, p<0.001) ;-4-0$ s vs. $4-8$ s $(z=-3.206$, $p<0.001)$ ).

- High band (HB) power

IPD patients in the OFF state exhibited a significantly greater power than healthy subjects in the pre-stimulus condition ( $\mathrm{C}$ vs. IPD OFF) $(-4-0 \mathrm{~s}$ : $U=56.0 ; z=-3.00 ; p=0.002)$. This observation remains true immediately after the perturbation (C vs. IPD OFF (0-4 s: $U=59.0 ; z=-2.90 ; p=0.003)$ ), which was also perceived in comparison between ON and OFF states (IPD OFF vs. IPD ON (0-4 s: $p=0.007)$ ).

An abrupt increase of power took place in the first $4 \mathrm{~s}$ after the visual perturbation $(-4-0$ vs. $0-4 \mathrm{~s})$ : (control $(z=-2.94 ; p=0.002)$, $\operatorname{IPD} \operatorname{OFF}(z=-2.79 ; p=0.003)$, IPD ON $(z=-1.874, p=0.064)$ ). Albeit IPD patients in the OFF state also had an increase of power $(-4-0 \mathrm{~s}$ vs. $4-8=s):(z=-2.172, p=0.029)$, it was not as striking as in healthy subjects and IPD in the ON state, specialy at the $4-8 \mathrm{~s}$ interval $(-4-0$ vs. $4-8 \mathrm{~s}$ : control $(z=-3.260, p<0.001)$ IPD ON $(z=-3.408$, $p<0.001)$ ). In fact, the constant increase of the HB power in IPD patients in the ON state is observed during the whole post-stimulus time interval (Fig. 5) (0-4 vs. 4-8 s: $(z=-2.101, p=0.035)$ ). 


\section{Discussion}

IPD patients, besides a motor output disorder, have also sensory and perceptual impairments, rendering them more vulnerable to misjudgments of sensory feedback [48]. Postural control and other cognitive processing share cognitive resources, and performance of postural tasks is often impaired by a secondary cognitive task. Individuals who have limited cognitive processing due to neurological impairments, such as IPD, may use more of their available cognitive processing to control posture [49], increasing their susceptibility to falls. Moreover, there is growing scientific evidence that CPA in reaction to external perturbations are compromised in IPD $[2,17]$. Therefore, we hypothesized that a moving immersive virtual reality environment would be a useful tool for analyzing CPA in response to visual perturbations on healthy subjects in comparison to IPD patients, and further understand the role of levodopa and thereafter the underlying mechanisms of corrective postural adjustments.

Stiffer systems, as those representing subjects with IPD, are characterized by frequent and rapid adjustments of COM position $[19,11]$. Patients with IPD in the ON state increased their sway velocity, magnifying the difference comparatively to healthy subjects (Table 2). Our results are in agreement with this finding since IPD swayed at a faster velocity than healthy subjects. As shown in Fig. 2, prominent differences between groups occurred mainly in the vertical direction (Z-axis). CPA on the vertical axis probably reflects a human mechanism of maintaining balance by varying the height of COM through knees and trunk bending. In this respect, levodopa may have facilitated this postural control mechanism by reducing muscular stiffness and rigidity. However, as further discussed on study limitations, the clear instruction to maintain a quite stance and use knee-bending as a corrective strategy, may have potentially played a greater role in observing prominent differences in CPAs along the vertical axis. The role of levodopa in balance maintaining is not straightforward and investigations concerning postural instability in subjects with IPD that depend on quiet stance features, often produce discrepant results. Some research suggests that levodopa improves some balance abnormalities but worsens the others [50,51]. In the ON state, IPD patients may present larger and faster sway just because levodopa reduces rigidity without improving control of posture, or because subclinical dyskinesia increases body motion [19].

Higher velocities and displacements of postural adjustments may just reflect the mechanical consequence to control the COM. However, different central postural control mechanisms may be continuously and dynamically taking place and therefore TDF analysis may provide further information on the postural reaction and susceptibility to visual perturbation. Since, we did not find distinct peaks in distinct bands due to a particular event, we suggest that all frequency components have an active role in standing balance. However, control mechanisms can actuate on specific frequency bands by switching between stabilizing (low) and corrective (high) processes. In our study, TFD analysis of vertical acceleration revealed that compensatory postural adjustments, in response to visual perturbation, were taking place in two distinct bands: a low $(<1.5 \mathrm{~Hz})$ and a high frequency $(>1.5 \mathrm{~Hz})$ band. This meets the findings of other studies, which demonstrated that postural control takes place in two distinct time scales: a fast (high frequency) open-loop control and a slower (low frequency) corrective feedback-based control [1]. LB components seem to be largely dictated by the inertial properties of the oscillating mass of the subject. In contrast, HB oscillatory components of sway are more likely to represent the lump sum of irregular, voluntary and involuntary muscle activity and multisensory feedback integration [52]. Moreover, performance on cognitive tasks has been shown to be only influenced by the variability of fast sway components [35].
In our study, the dissonance of the visual input with the somatosensory perceptions (proprioceptive and vestibular), provoked by VR, induced CPA. These CPA were most evident immediately after visual environment scenario change but carried on till $8 \mathrm{~s}$ later. The immediate period after visual environment change may be the most important for major compensatory postural correction. After that other mechanisms, like reaction to minor details of the visual environment, may play their hand. These postural corrections seem to happen predominantly on the highfrequency spectra, as illustrated in Fig. 5. Immediately after the environment visual scenario change, IPD patients in the OFF state significantly reacted with higher power on HB than healthy subjects. However, IPD patients in the OFF state presented a different trend in comparison to Controls and IPD patients in the ON state. After the visual perturbation, IPD in the ON state had a similar trend to respond to visual perturbation in the HB similar to healthy subjects, which was even more prominent in the last interval.

As previously stated postural control that takes place in the $\mathrm{HB}$ probably reflects a more central cognitive and volitional mechanism of postural control [35,1], A recent study using VR and gait analysis on IPD, provided evidence that cognitive dysfunction, such as anxiety, interfered with proper information processing. On this study, dopaminergic medication improved utilization of sensory feedback in stressful situations by reducing anxiety and/or improving resource allocation [53]. Our findings (summarized in Table 3) corroborate the benefit of levodopa, as IPD in the OFF state presented more dependence of CPAs on the HB, further attenuated after levodopa in the ON state.

Healthy subjects maintained their LB frequencies power essentially unchanged, even after the visual perturbation. On the other hand, an abrupt increase of high frequencies power occurred after the stimulus onset. Lower frequencies probably reflect a more mechanical automatic oscillation mechanism of stabilization and posture control, dependent on the stiffness of the musculotendinous structure. Fig. 4 suggests that in the ON state, previous to visual perturbation, CPAs in the LB are more pronounced in comparison with the other groups. Probably, the inherent higher instability on IPD requires a greater actuation of continuous restoring and compensatory forces, which is provided by slow acceleration components. This strategy, achieved with levodopa treatment, is reflected by a significant shift of IMF towards the low frequencies in subjects with IPD in the ON state. These slow oscillations might indicate an improvement of muscle rigidity of IPD patients in the ON state. Without levodopa, IPD patients exhibited a need for a rapid increase of corrective adjustments on the $\mathrm{HB}$, and afterwards a need for greater restoring effect provided by the LB. This probably reflects a higher susceptibility to the visual perturbation and need to produce corrective adjustments to external perturbations in the OFF state of IPD. Moreover, the trend to LB CPAs in the OFF state may indeed reflect the mechanical constraint of muscular rigidity. The passive stiffness of the musculotendinous structure of the human body stands out when maintaining quiet erect posture (as still as possible), either for the muscle completely relaxed or with muscle tone. The passive stiffness acts similar to an "elastic" opposed to the torque of gravitational force, which has the tendency to cause a forward fall of the body. Although the estimative of the contribution of the restoring torque due to the passive stiffness varies widely in the literature, it is estimated that this torque ranges about $65 \%$ to $90 \%$ from the magnitude of the gravitational torque [54,55]. Therefore, more than half of the torque responsible for maintaining our erect posture would be generated by a purely passive component, independent of the direct participation of the nervous system [56]

On previous literature, using a surface perturbation platform and electromyographic (EMG) analyzes of the automatic gastrocnemius or tibialis anterior muscle responses, it was shown that IPD patients had slower sensoriomotor responses and more difficulty in 
using cognitive set to modify their responses [15]. In this study levodopa medication did not improve the Parkinson subjects' ability to change set quickly [15]. In another study, with similar methodology, levodopa medication improved tonic background postural tone but further weakened automatic postural responses to external displacements [14]. On a multidirectional perturbations surface reactive forces and EMG trunk and limb analyzes, it was shown that IPD subjects, evaluated in OFF state, present excess activation of antagonist muscles across many perturbation directions. Their limited ability to modify postural muscle activity with changes in stance width, from wide to narrow stance, supported the hypothesis that the basal ganglia are important for optimizing muscle activation patterns by quickly switching motor patterns when the task or environment changes [17].

Locomotion and postural control involve cognitive volitional initiation and automatic controlled movement processes, such as the adjustment of postural muscle tone and rhythmic limb movements. Information processing between the basal ganglia, the cerebellum, and the brainstem may enable automatic regulation of muscle tone and rhythmic limb movements in the absence of conscious awareness. The basal ganglia play an important role in the control of axial tone, postural response amplitude, and interpretation of somatosensory information [57] . Thus, postural abnormalities in early IPD are not surprising. According to our results, this automatic postural control is responsive to levodopa and happens in a lower frequencies band. Intentional postural and gait modification, e.g., reacting to a visual obstacle and environment, requires motor programming in the premotor cortices. These motor programs utilize one's bodily information, such as the body schema, which is preserved and updated in the temporoparietal cortex. The motor programs are transmitted to the brainstem by the corticoreticulospinal system, so that one's posture is anticipatorily controlled [57]. According to our results, these volitional postural responses happen in a higher frequencies band.

\section{Study limitations}

Studies that have shown distinct timescale mechanisms for postural control, a low and a fast, have defined cut-offs of $0.3 \mathrm{~Hz}$ for COP position data $[1,35]$. Accelerometer data requires a new analysis in a range of frequencies from 0.3 to $3.5 \mathrm{~Hz}$ to determine which frequency would yield a proper discrimination between slow and fast components of postural sway. In our study, to distinguish between low- and high-frequency domains, a band separation at $1.5 \mathrm{~Hz}$ was chosen, as upon a visual inspection it appeared to yield representative and discriminative results. Nevertheless, this decision was purely subjective and should be explored further. Retrospective analysis of our clinical records revealed that 8 out of 15 IPD patients had at least one minor fall without injury, on the past two years. Our small sample impaired further robust statistical inference. As falls are very prevalent and important issue on IPD, further studies, with larger samples, are needed for kinematic analysis of CPAs to be used as an additional instrument to predict and differentiate different risks of falls. The clear instruction for subjects to maintain a fixed posture, with feet together position, and use kneebending as a corrective strategy, may have potentially played a greater role in observing prominent differences in CPAs along the vertical axis (z-axis). Had they not been given these specific instructions upfront, the CPAs may have shown different patterns along different axes. As so, our methodology, albeit allowing better standardization between subjects and justifying our time frequency analyzes on the vertical axis (z-axis), has to be taken with caution as may not reflect the real life scenario, where a forward or lateral step correction, or use of lateral muscles, usually happen on freely open space. Furthermore, CPAs have shown to be higher in lateral muscles, especially on older, fallers and non-fallers, patients [5]. Nevertheless, using a narrow stance evaluation is very similar to the normal IPD progression, as patients with late-stage PD tend to stand and to walk with narrower and narrower stance width, and evaluating patients on narrow stance may further elicit differences to controls $[58,17]$, as shown in our study.

\section{Conclusion}

In summary, our results showed that a VR model, which produced a sensorial dissonance between a changing visual environment in contrast to static sensorial inputs, induced significant compensatory postural adjustments on healthy subjects and IPD patients. Different postural adjustment mechanisms took place in LB and HB frequencies. LB aspects of CPAs are due to inertial properties of the oscillating mass, and, therefore, more dependent on the effect of levodopa on stiffness and rigidity. The HB oscillatory components of CPAs are more likely to represent multisensory feedback integration and a reaction to the ongoing visual input-changing scenario. IPD patients in the OFF state exhibited an abnormal compensatory response to the visual stimulus. Levodopa allowed these patients to perform with similarly to healthy subjects and lowered the $\mathrm{HB}$ response immediately after the visual perturbation. On the other hand, levodopa compensated the inherent instability of the IPD condition through an increased stabilizing force provided by low frequencies. This effect probably reflects a decrease of muscular stiffness and rigidity. Despite the overall larger and faster sway in the ON state, levodopa seems to improve CPAs in patients with IPD. Further studies are needed to evaluate CPA on VR paradigms with more open-space free movements, and even in real life scenarios.

\section{Acknowledgments}

The Center ALGORITMI was funded by the FP7 Marie Curie ITN Neural Engineering Transformative Technologies (NETT) project. The authors have no conflicts of interest to report.

\section{References}

[1] M.R.C. Heuvel, R. Balasubramaniam, A. Daffertshofer, A. Longtin, P.J. Beek, Delayed visual feedback reveals distinct time scales in balance control, Neurosci. Lett. 452 (1) (2009) 37-41.

[2] N. Kanekar, A.S. Aruin, Aging and balance control in response to external perturbations: role of anticipatory and compensatory postural mechanisms, AGE 36 (2014) 1067-1077.

[3] S. Park, F.B. Horak, A.D. Kuo, Postural feedback responses scale with biomechanical constraints in human standing, Exp. Brain Res. 154 (4) (2004) 417-427.

[4] A.V. Alexandrov, A.A. Frolov, F.B. Horak, P. Carlson-Kuhta, S. Park, Feedback equilibrium control during human standing, Biol. Cybern. 93 (5) (2005) 309-322.

[5] R. Claudino, E. Santos, M.J. Santos, Compensatory but not anticipatory adjustments are altered in older adults during lateral postural perturbations, Clin. Neurophysiol. 124 (8) (2013) 1628-1637.

[6] J. Massion, Movement, posture and equilibrium: Interaction and coordination, Prog. Neurobiol. 38 (1) (2003) 35-56.

[7] T.E. Prieto, J.B. Myklebust, R.G. Hoffmann, E.G. Lovett, B.M. Myklebust, Measures of postural steadiness: differences between healthy young and elderly adults, IEEE Trans. Biomed. Eng. 43 (9) (1996) 956-966.

[8] R.J. Peterka, P.J. Loughlin, Dynamic regulation of sensorimotor integration in human postural control, J. Neurophysiol. 91 (1) (2003) 410-423.

[9] M.V. Diest, C.J. Lamoth, J. Stegenga, G.J. Verkerke, K. Postema, Exergaming for balance training of elderly: state of the art and future developments, J. NeuroEng. Rehab. 10 (1) (2013) 101

[10] J.-B. Mignardot, O. Beauchet, C. Annweiler, C. Cornu, T. Deschamps, Postural sway, falls and cognitive status: a cross-sectional study among older adults, J. Alzheimer's Dis. 41 (2014) 431-439.

[11] M. Mancini, F.B. Horak, C. Zampieri, P. Carlson-Kuhta, J.G. Nutt, L. Chiari, Trunk accelerometry reveals postural instability in untreated Parkinson's disease, Parkinsonism Related Disorders 17 (7) (2011) 557-562.

[12] Y.A.M. Grimbergen, A.D. Speelman, M.A. van der Marck, Y. Schoon, B.R. Bloem, Gait, postural instability, and freezing, in: Parkinson's Disease: Non-Motor and Non-Dopaminergic Features, Blackwell Publishing Ltd., Oxford, UK, 2011, pp. 261-373. 
[13] A. Conte, N. Khan, G. Defazio, J.C. Rothwell, A. Berardelli, Pathophysiology of somatosensory abnormalities in Parkinson disease, Nat. Rev. Neurol.y 9 (12) (2013) 687-697.

[14] F.B. Horak, J. Frank, J. Nutt, Effects of dopamine on postural control in parkinsonian subjects: scaling, set, and tone, J. Neurophysiol. 75 (6) (1996) 2380-2396.

[15] R.K.Y. Chong, F.B. Horak, M.H. Woollacott, Parkinson's disease impairs the ability to change set quickly, J. Neurol. Sci. 175 (1) (2015) 57-70.

[16] M. Mancini, C. Zampieri, P. Carlson-Kuhta, L. Chiari, F.B. Horak, Anticipatory postural adjustments prior to step initiation are hypometric in untreated Parkinson's disease: an accelerometer-based approach, Eur. J. Neurol. 16 (9) (2009) 1028-1034.

[17] D. Dimitrova, Postural muscle responses to multidirectional translations in patients with Parkinson's disease, J. Neurophysiol. 91 (1) (2003) 489-501.

[18] D.H. Romero, G.E. Stelmach, Changes in postural control with aging and Parkinson's disease, IEEE Eng. Med. Biol. Mag. 22 (2) (2003) 27-31.

[19] L. Rocchi, L. Chiari, F.B. Horak, Effects of deep brain stimulation and levodopa on postural sway in Parkinson's disease, J. Neurol. Neurosurg. Psychiatry 73 (3) (2002) 267-274.

[20] C. Maurer, T. Mergner, J. Xie, M. Faist, P. Pollak, C.H. Lücking, Effect of chronic bilateral subthalamic nucleus (STN) stimulation on postural control in Parkinson's disease, Brain 126 (2003) 1146-1163.

[21] M. Contin, R. Riva, A. Baruzzi, F. Albani, S. Macri, P. Martinelli, Postural stability in Parkinson's disease: the effects of disease severity and acute levodopa dosing, Parkinsonism Related Disorders 2 (1) (1996) 29-33.

[22] M.F. Gago, V. Fernandes, J. Ferreira, H. Silva, M.L. Rodrigues, L. Rocha, E. Bicho, N. Sousa, The effect of levodopa on postural stability evaluated by wearable inertial measurement units for idiopathic and vascular Parkinson's disease, Gait Posture (2014)

[23] L.M. Hall, S.G. Brauer, F. Horak, P.W. Hodges, The effect of Parkinson's disease and levodopa on adaptation of anticipatory postural adjustments, Neuroscience 250 (2013) 483-492.

[24] M.F. Gago, V. Fernandes, J. Ferreira, H. Silva, L. Rocha, E. Bicho, N. Sousa, Postural stability analysis with inertial measurement units in Alzheimer's disease, Dement. Geriatr. Cogn. Disorders Extra (2014) 22-30.

[25] M.A. Riley, S. Clark, Recurrence analysis of human postural sway during the sensory organization test, Neurosci. Lett. $342(1-2)(2003) 45-48$.

[26] T.A. Stoffregen, R.J. Pagulayan, B.G. Bardy, L.J. Hettinger, Modulating postural control to facilitate visual performance, Hum. Mov. Sci. 19 (2) (2015) 203-220.

[27] P.L. Weiss, E. Keshner, M.F. Levin, Vision, perception, and object manipulation in virtual environments, in: Virtual Reality Technologies for Health and Clinical Applications, Springer, New York, 2014, pp. 47-70.

[28] K. Kobayashi, H. Fushiki, M. Asai, Y. Watanabe, Head and body sway in response to vertical visual stimulation, Acta Oto-laryngologica 125 (8) (2005) $858-862$.

[29] K. Dokka, R.V. Kenyon, E.A. Keshner, Influence of visual scene velocity on segmental kinematics during stance, Gait \& Posture 30 (2) (2009) 211-216.

[30] M.J. Mathie, A.C. Coster, N.H. Lovell, B.G. Celler, Accelerometry: providing an integrated, practical method for long-term, ambulatory monitoring of human movement, Physiol. Meas. 25 (2) (2004) R1-20.

[31] L. Palmerini, L. Rocchi, S. Mellone, F. Valzania, L. Chiari, Feature selection for accelerometer-based posture analysis in Parkinson's disease, IEEE Trans. Inf. Technol. Biomed. 15 (3) (2011) 481-490.

[32] T. Watanabe, H. Saito, E. Koike, K. Nitta, A preliminary test of measurement of joint angles and stride length with wireless inertial sensors for wearable gait evaluation system, Comput. Intell. Neurosci. (2011) 1-12.

[33] J.P. Carroll, W. Freedman, Nonstationary properties of postural sway, J. Biomech. 26 (4-5) (1993) 409-416.

[34] T. Shumann, M.S. Refern, J.M. Furman, A. El-Jaroudi, L.F. Chaparro, Time-frequency analysis of postural sway. J. Biomech. 28 (5) (1995) 603-607.

[35] T.T. Yeh, J. Boulet, T. Cluff, R. Balasubramaniam, Contributions of delayed visual feedback and cognitive task load to postural dynamics, Neurosci. Lett. 481 (3) (2010) 173-177.
[36] C.H. Williams-Gray, T. Foltynie, C.E.G. Brayne, T.W. Robbins, R.A. Barker, Evolution of cognitive dysfunction in an incident Parkinson's disease cohort, Brain 130 (7) (2007) 1787-1798.

[37] S. Freitas, M.R. Simões, L. Alves, I. Santana, Montreal cognitive assessment (MoCA): normative study for the Portuguese population, J. Clin. Exp. Neuropsychol. 33 (9) (2011) 989-996.

[38] J.A. Afonso, H.D. Silva, P. Macedo, L.A. Rocha, An enhanced reservation-based MAC protocol for IEEE 802. 15. 4 networks, Sensors 11 (4) (2011) 3852-3873.

[39] A. Al-Jawad, A. Barlit, M. Romanovas, M. Traechtler, Y. Manoli, The use of an orientation Kalman filter for the static postural sway analysis, APCBEE Proc. 7 (2013) 93-102.

[40] M.E. Williams, Geriatric Physical Diagnosis: A Guide to Observation and Assessment, NC: McFarland \& Co., Jefferson, 2008.

[41] S.S. Haykin, Time-Frequency Signal Analysis, in: Advances in spectrum analysis and array processing, Cliffs, N.J., Prentice Hall, Englewood, 1991, pp. 418-517.

[42] A. Papandreou-Suppappola, Time-frequency processing: tutorial on principles and practice, in: Applications in Time-Frequency Signal Processing, CRC Press, Boca Raton, 2003, pp. 1-84.

[43] P. Loughlin, J. Pitton, B. Hannaford, Approximating time-frequency density functions via optimal combinations of spectrograms, IEEE Signal Process. Lett. 1 (12) (1994) 199-202.

[44] P.J. Loughlin, J.W. Pitton, L.E. Atlas, Construction of positive time-frequency distributions, IEEE Trans. Signal Process. 42 (10) (1994) 2697-2705.

[45] R. Martinez-Mendez, M. Sekine, T. Tamura, Postural sway parameters using a triaxial accelerometer: comparing elderly and young healthy adults, Comput. Methods Biomech. Biomed. Eng. 15 (9) (2012) 899-910.

[46] P.J. Loughlin, M.S. Redfern, Spectral characteristics of visually induced postural sway in healthy elderly and healthy young subjects, IEEE Trans. Neural Syst. Rehab. Eng. 9 (1) (2001) 24-30.

[47] M. Ferdjallah, G.F. Harris, J.J. Wertsch, Instantaneous postural stability characterization using time-frequency analysis, Gait Posture 10 (2) (1999) $129-134$.

[48] K.A.E. Martens, Q.J. Almeida, Dissociating between sensory and perceptual deficits in PD: More than simply a motor deficit, Mov. Disorders 27 (3) (2011) 387-392.

[49] F.B. Horak, Postural orientation and equilibrium: what do we need to know about neural control of balance to prevent falls? Age Ageing 35 (S2) (2006) ii7-ii11.

[50] N.I. Bohnen, R. Cham, Postural control, gait, and dopamine functions in Parkinsonian movement disorders, Clin. Geriatr. Med. 22 (4) (2006) 797-812.

[51] F.J. Revilla, T.R. Larsh, A. Mani, A.P. Duker, C. Cox, P. Succop, M. Gartner, C.J. Tejada, A. Bhattacharya, Effect of dopaminergic medication on postural sway in advanced Parkinson's disease, Front. Neurol. 4 (202) (2013).

[52] M.I. Jordan, D.M. Wolpert, Computational motor control, in: The Cognitive Neurosciences, Cambridge, MIT Press, MA, 1999, pp. 601-620.

[53] K.A.E. Martens, C.G. Ellard, Q.J. Almeida, Virtually-induced threat in Parkinson's: dopaminergic interactions between anxiety and sensory-perceptual processing while walking, Neuropsychologia (2015).

[54] M. Casadio, P.G. Morasso, V. Sanguineti, Direct measurement of ankle stiffness during quiet standing: implications for control modelling and clinical application, Gait Posture 21 (4) (2005) 410-424.

[55] I.D. Loram, M. Lakie, Direct measurement of human ankle stiffness during quiet standing: the intrinsic mechanical stiffness is insufficient for stability, J. Physiol. 545 (3) (2002) 1041-1053.

[56] B.R. Bloem, J.M. Hausdorff, J.E. Visser, N. Giladi, Falls and freezing of gait in Parkinson's disease: a review of two interconnected, episodic phenomena, Mov. Disorders 19 (8) (2004) 871-884.

[57] K. Takakusaki, Neurophysiology of gait: from the spinal cord to the frontal lobe, Mov. Disorders 28 (11) (2013) 1483-1491.

[58] J.G. Nutt, F.B. Horak, Gait and balance disorders, in: W. Koller (Ed.), Movement Disorders: Neurologic Principles and Practice, McGraw-Hill, 1996, pp. 649-660. 